\title{
Unilateral pedicle screw instrumentation for minimally invasive transforaminal lumbar interbody fusion
}

\author{
Will F. Beringer, D.O., AND Jean-Pierre Mobasser, M.D. \\ Indianapolis Neurosurgical Group, Indianapolis, Indiana
}

\begin{abstract}
Object. Clinical and radiographic results were assessed to determine the clinical outcomes and fusion rate in eight consecutive patients selected for minimally invasive transforaminal lumbar interbody fusion (TLIF) in which a percutaneous pedicle screw system was used unilaterally.

Methods. Eight patients underwent one-level, minimally invasive TLIF in which a percutaneous pedicle screw system was used only on the side where facets were removed for interbody access. Clinical, economic, functional, and radiographic data were recorded preoperatively and at 6 months postoperatively. The mean 6-month change in the modified Prolo Scale score was 7, and osseous interbody bridging assessed on coronal and sagittal computerized tomography studies was seen in all patients.

Conclusions. The use of unilateral percutaneous pedicle screw instrumentation for the minimally invasive TLIF procedure provides excellent clinical results and is an option in selected patients.
\end{abstract}

\section{KEY WORDS • pedicle screw • minimally invasive spinal fusion • transforaminal lumbar interbody fusion}

$\mathrm{W}$ HEN it was described by Harms and Rollinger, 5 TLIF was an innovative improvement in posterior surgical approaches to the disc space for interbody arthrodesis. This procedure has continued to evolve as clinical researchers explore less invasive ways to achieve the same surgical goals with mini-open and minimally invasive approaches. ${ }^{3,4,11}$ The pursuit of less invasive approaches that has led to the development and refinement of the TLIF procedure is driven by the desire for better clinical results, less postoperative pain for patients, shorter operating room times and duration of hospital stay, and reduced overall cost. ${ }^{3,15,17}$

Recent innovations in percutaneous pedicle screw instrumentation and access to the disc space with tubular retractors have allowed the goals of TLIF to be accomplished with minimal trauma to the paraspinal muscles and osseous and ligamentous architecture. ${ }^{3}$ Moreover, the offlabel use of osteoinductive rhBMP-2 in addition to autograft has yielded faster interbody arthrodesis than with autograft-packed cages alone in patients undergoing the TLIF procedure. ${ }^{10}$

With the goal of less invasive surgery in mind, several researchers have tested whether bilateral pedicle screw instrumentation is necessary for internal fixation while posterolateral arthrodesis is being established. ${ }^{7,16}$ The results of these studies have shown that unilateral pedicle screw

Abbreviations used in this paper: $\mathrm{CT}=$ computerized tomography; DDD = degenerative disc disease; rhBMP-2 = recombinant human bone morphogenetic protein-2; TLIF = transforaminal lumbar interbody fusion. fixation can be as effective as the bilateral method in posterolateral spinal fusion. Although there has been no study of the clinical efficacy of unilateral pedicle screw instrumentation for TLIF, Harris and coworkers ${ }^{6}$ have used an in vitro cadaver model to investigate the flexibility of various TLIF constructs. The question they raised is how much construct stiffness is necessary to establish interbody arthrodesis, given the innovations in minimally disruptive TLIF and the demonstrated efficacy of rhBMP-2 in establishing interbody arthrodesis. In this paper, we prospectively followed a series of eight patients selected for minimally invasive TLIF and unilateral pedicle screw instrumentation to assess their fusion rate and clinical outcomes.

\section{Clinical Material and Methods}

Eight patients requiring TLIF, in whom relatively normal facets were demonstrated on preoperative neuroimaging, were selected for unilateral percutaneous pedicle screw fixation. Their imaging studies, hospital charts, and office records were analyzed for data collection. Telephone interviews supplemented data recorded at postoperative office visits. The descriptions of these patients are presented in Table 1. Four patients were treated for multiple recurrent disc herniations, and four were treated for symptomatic DDD. All patients experienced a combination of mechanical back pain and lumbar radiculopathy; two had filed Workers' Compensation claims. In the patients with symptomatic DDD in our study, at least 9 months of conservative management had failed, and concordant pain was eli- 
TABLE 1

Descriptive statistics of patients with preoperative and 6-month postoperative modified Prolo Scale scores*

\begin{tabular}{|c|c|c|c|c|c|}
\hline \multirow{2}{*}{$\begin{array}{l}\text { Case } \\
\text { No. }\end{array}$} & \multirow[b]{2}{*}{ Age (yrs) } & \multirow[b]{2}{*}{ Diagnosis } & \multirow[b]{2}{*}{ Level Fused } & \multicolumn{2}{|c|}{$\begin{array}{c}\text { Prolo } \\
\text { Scale Score }\end{array}$} \\
\hline & & & & Preop & Postop \\
\hline 1 & 45 & recurrent HNP & L5-S1 & 11 & 19 \\
\hline 2 & 35 & DDD & L5-S1 & 12 & 18 \\
\hline 3 & 43 & DDD & L5-S1 & 11 & 19 \\
\hline 4 & 58 & recurrent HNP & L2-3 & 11 & 18 \\
\hline 5 & 40 & DDD/HNP & L5-S1 & 6 & 15 \\
\hline 6 & 58 & $\begin{array}{l}\text { redo for foraminal } \\
\text { stenosis/DDD }\end{array}$ & L4-5 & 12 & 19 \\
\hline 7 & 63 & HNP & L4-5 & 11 & 15 \\
\hline 8 & 53 & recurrent HNP & L5-S1 & 11 & 19 \\
\hline
\end{tabular}

* All patients had attained fusion at 6-month follow-up evaluation. Abbreviation: $\mathrm{HNP}=$ herniated nucleus pulposus

cited on provocative discography, with no pain found at the adjacent control level.

All patients had followed an extensive conservative care regimen before undergoing surgical intervention. Their care consisted of a combination of the following: physical therapy, medications, nerve blocks, epidural injections of steroid agents, activity modification, weight loss, diet modification, and external bracing (Fig. 1).

Fusion was assessed using thin-cut lumbar CT scans with reformatted images that were obtained at the 6-month follow-up evaluation. Patients with solid osseous trabeculations bridging across the interspace were considered to have attained fusion. Assessment of functional outcome was completed using a modified Prolo scale (Table 2).12,14

\section{Surgical Technique}

After induction of general endotracheal anesthesia, patients were positioned prone on a radiolucent Jackson table (OSI, Union City, CA). A 3-cm incision was made 4.5 to $5 \mathrm{~cm}$ off the midline, over the side chosen for the TLIF approach. A K-wire was passed down through the fascia, aided by direct fluoroscopic vision. Sequential dilators were passed over the K-wire, and a 22-mm-diameter tubular port of appropriate length was docked on the facet joint to be removed. With the aid of fluoroscopic guidance, osteotomes were used to chisel out the facet and lateral aspect of the lamina. This bone was removed and kept for use as an autograft during the interbody fusion. The thecal sac and traversing nerve root were identified. A thorough discectomy and endplate preparation were performed using Pyrametrix Advance Instrument Set (Medtronic Sofamor Danek, Memphis, TN).

Once the distraction and endplate preparation were complete, morcellized autograft rolled in an rhBMP-2treated sponge was placed in the disc space and moved to the contralateral side. This was followed by driving in an appropriately sized Capstone implant (Medtronic Sofamor Danek) filled with autograft and an rhBMP-2-treated sponge, with the device and its contents being inserted obliquely into the intervertebral space. Lateral fluoroscopy was used for guidance during these procedures. Another autograft rolled in an rhBMP-2-treated sponge was then inserted ipsilateral to the graft.

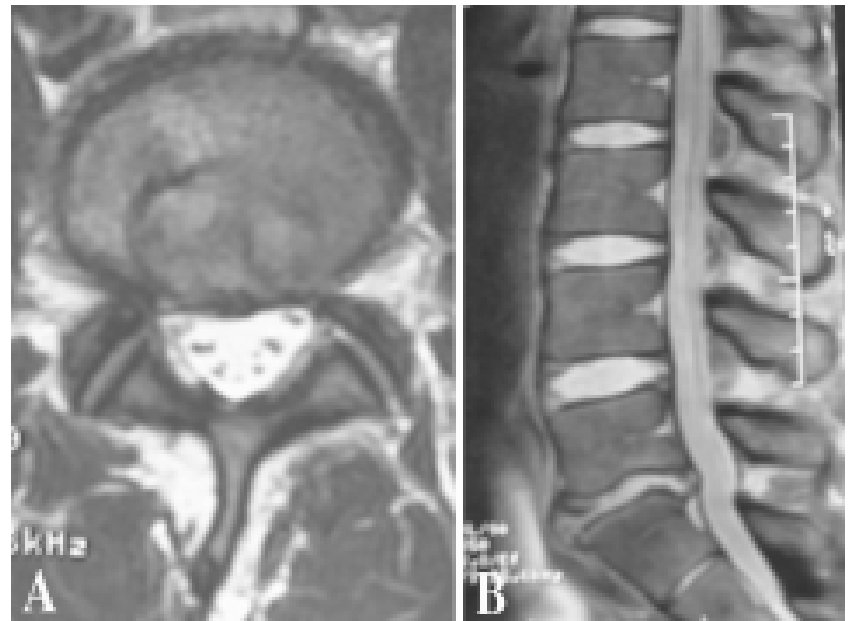

FIg. 1. Axial (A) and sagittal (B) $\mathrm{T}_{2}$-weighted magnetic resonance images of a degenerated L5-S1 disc in a 43-year-old man in whom conservative management failed.

Once the interbody discectomy and arthrodesis were completed, the 22-mm METRx tubular port was removed and SEXTANT instrumentation (Medtronic Sofamor Danek) was inserted through the same 3-cm incision. The technical report on SEXTANT instrumentation published by Foley and Gupta $^{2}$ provides a description of this portion of the procedure.

\section{Results}

The mean follow-up duration was 9 months, with a range of 6 to 12 months. There were no conversions to open surgery from the minimally invasive approach. The mean operating time was 160 minutes (range 145-180 minutes), and the mean estimated blood loss was $100 \mathrm{ml}$ (range 50-200 ml). The mean duration of hospital stay was 1.6 days (range 1-3 days). All but one patient with preoperative radiculopathy attained resolution of their symptoms postoperatively, and all patients with mechanical low-back pain noted improvement of this symptom. The mean modified Prolo Scale score was 11 (range 6-12) preoperatively, whereas the mean score 6 months postoperatively was 18 (range 15-19).

There were no new postoperative radiculopathies or malpositioned pedicle screws. One patient requested removal of his unilateral percutaneous pedicle screw instrumentation because it caused muscle spasm and pain. His instrumentation was removed using a mini-open technique 6 months postoperatively, after bone fusion from the TLIF procedure was demonstrated. This patient's muscle pain had resolved by his 9-month postoperative visit. His 6-month modified Prolo Scale score was 18, and at 1 year it was 20.

At the 6-month postoperative follow-up assessment, all patients had solid bone fusions across the interspace. There was no sign of heterotopic ossification in any patient on follow-up imaging. No patient experienced migration of the intervertebral distracting implant (Figs. 2 and 3). 
TABLE 2

Modified Prolo Scale used for assessment at 6-month follow-up evaluation*

\begin{tabular}{cl}
\hline \hline \multicolumn{1}{c}{ Score } & \\
\hline pain & \\
P1 & unbearable \\
P2 & severe \\
P3 & moderate \\
P4 & mild \\
P5 & none \\
functional status & \\
F1 & total incapacity \\
F2 & can do activities at home \\
F3 & activities outside home w/ limitation \\
F4 & limitation w/ strenuous activities \\
F5 & able to do everything \\
economic status & \\
E1 & unable to do tasks around home \\
E2 & able to do tasks around home but unable to perform \\
& paid work \\
E3 & able to work at sedentary capacity \\
E4 & able to work at moderate capacity \\
E5 & able to work at heavy capacity or previous job \\
medication & none or occasional hydrocodone \\
M1 & 6-9 hydrocodone tablets or equivalent \\
M2 & regular NSAIDs or occasional hydrocodone \\
M3 & nonydrocodone tablets or equivalent \\
M4 & \\
M5 & \\
\hline
\end{tabular}

* NSAIDs = nonsteroidal antiinflammatory drugs.

\section{Discussion}

The TLIF procedure is a safe, reliable approach for interbody arthrodesis in selected patients. ${ }^{8,13,14}$ Fusion rates for open and minimally invasive TLIF procedures in which bilateral pedicle screw instrumentation is used have been noted to be 90 to $100 \% .^{3,8,10}$ Nevertheless, the mean blood loss for single-level open TLIF has been reported to be as much as $1612 \pm 1038 \mathrm{ml} .{ }^{14}$ The minimally invasive refinement of this technique improves safety and maintains good fusion outcomes, with a mean reported blood loss of $140 \mathrm{ml}$ (range $50-450 \mathrm{ml}$ ) and a mean duration of hospital stay of 1.9 days. ${ }^{3}$ Use of unilateral percutaneous pedicle screw instrumentation instead of bilateral pedicle screws further reduces the tissue injury and blood loss associated with this technique. We believe this caused less postoperative pain and translated to earlier mobilization and hospital discharge. Our patients had remarkable functional outcomes, with a mean modified Prolo Scale score of 18 at their 6-month follow-up evaluation. The mean improvement in the modified Prolo Scale score was 7. We attribute these results to our minimally invasive technique and our careful selection of surgical candidates.

Some authors have removed the posterior lateral lip of concave bone at the intervertebral space to achieve a flat endplate surface in preparation for driving in the cage. ${ }^{14}$ Alternatively, patients can be placed on a Wilson frame to help with intervertebral distraction and placement of an interbody cage. We found neither of these steps necessary because the biconcave shape of the self-distracting Capstone implant used allowed easy placement of the interbody cage after sequential distraction with the Capstone trials. The Capstone implant height ranged from
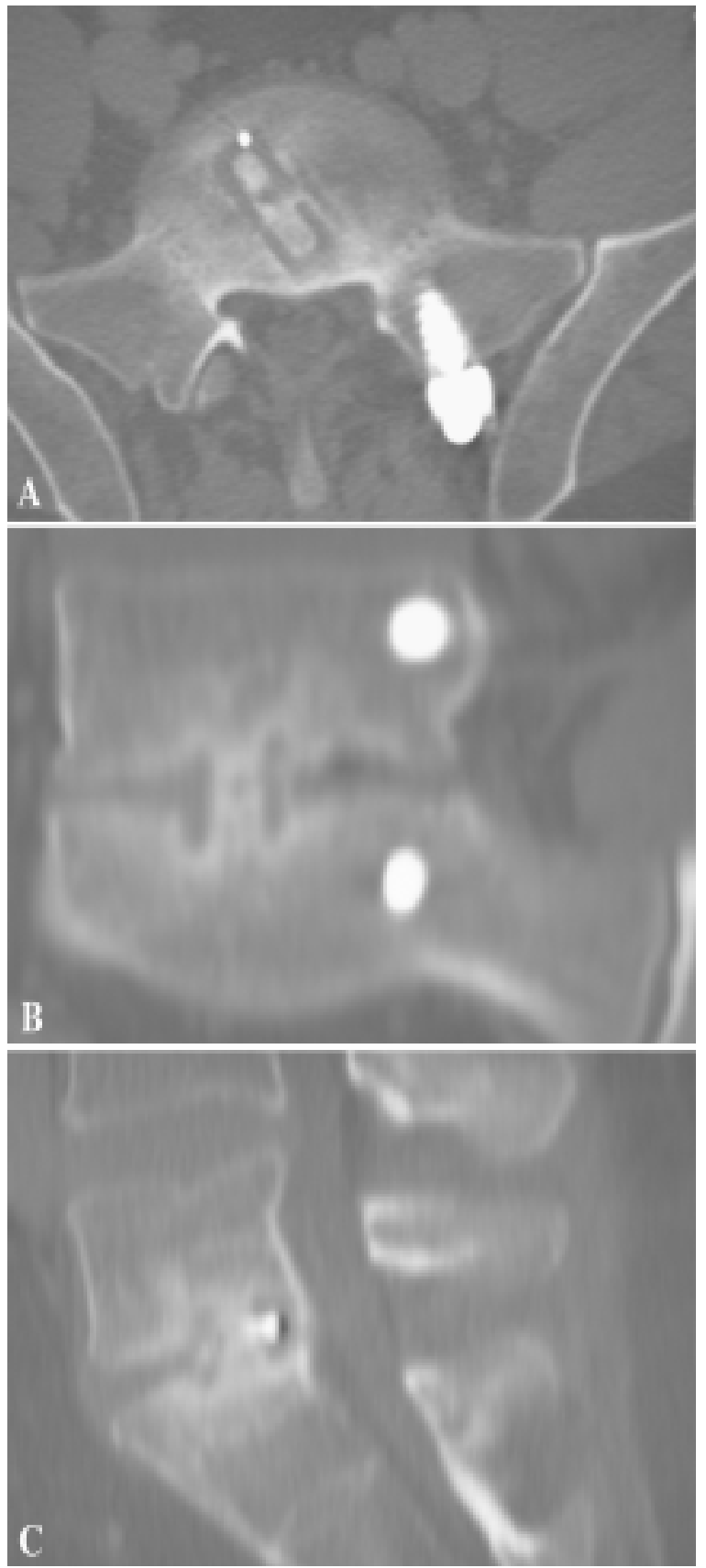

FIG. 2. Neuroimages obtained 6 months postoperatively. A: Axial CT scan demonstrating placement of a Capstone interbody cage with unilateral pedicle screw instrumentation. B: Coronal CT reconstruction demonstrating osseous interbody bridging and unilateral pedicle screw instrumentation. C: Sagittal CT reconstruction demonstrating osseous bridging inside and behind the interbody cage.

10 to $14 \mathrm{~mm}$. Selection of the cage size was based on fluoroscopic images that showed intervertebral height similar to adjacent disc space heights.

Osteobiological materials such as rhBMP-2 offer the possibility of increasing both fusion rates and the speed at which fusion occurs. ${ }^{9}$ Mummaneni, et al., ${ }^{10}$ studied 40 patients who had undergone open TLIF procedures and 


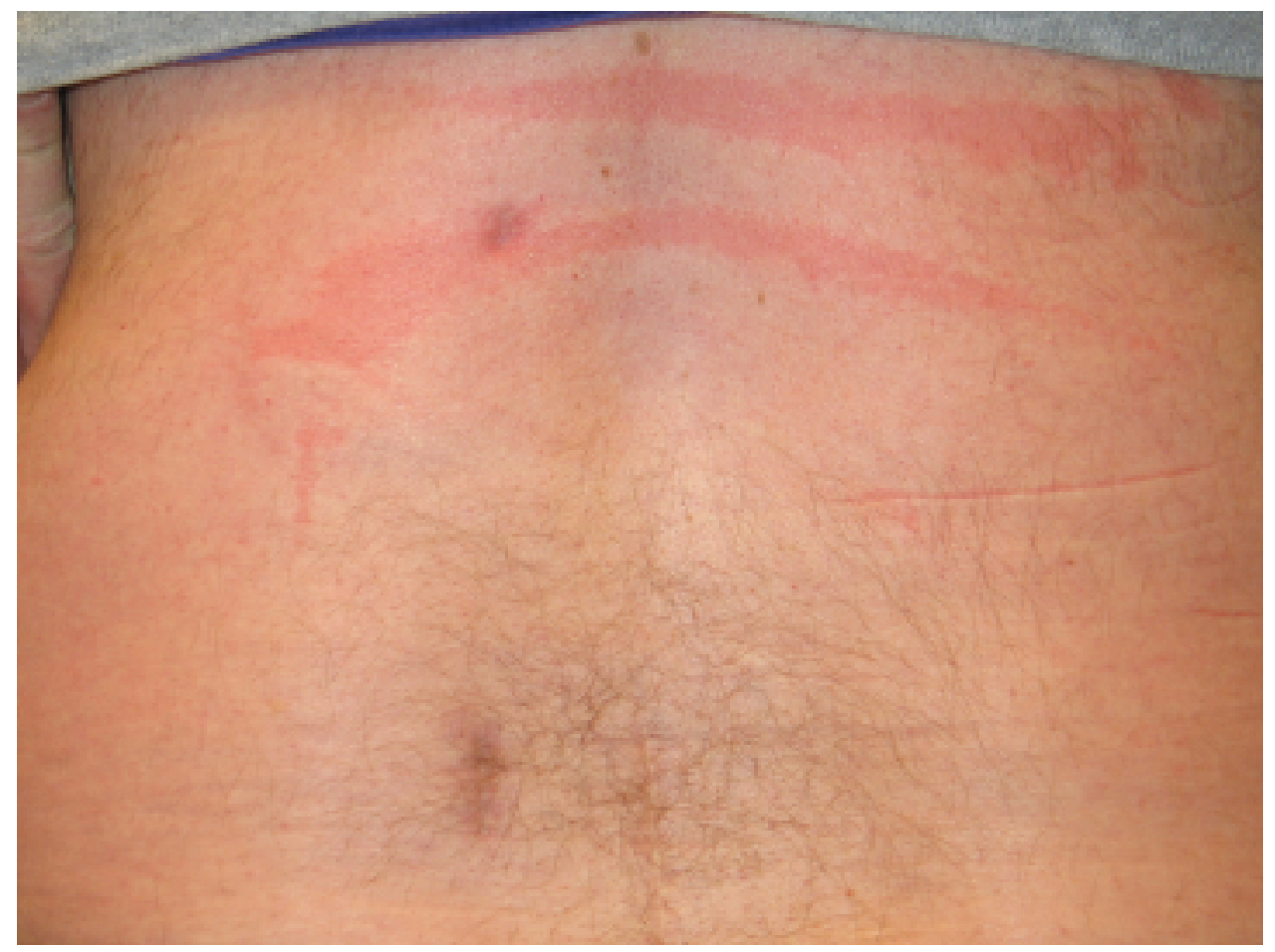

FIG. 3. Photograph obtained postoperatively showing the placement of the incision for the minimally invasive TLIF procedure and SEXTANT instrumentation $4.5 \mathrm{~cm}$ left of the midline. The original midline discectomy incision can also be seen.

compared the fusion rates of TLIF performed with iliac crest interbody autograft to that performed with iliac crest autograft plus rhBMP-2-treated sponges or local autograft plus rhBMP-2-treated sponges. Although the TLIF procedure performed with an interbody cage plus iliac crest autograft provided a $95 \%$ fusion rate at a mean of 6 months, no patient with complete follow up who had received rhBMP-2 experienced pseudarthrosis. The mean time to interbody fusion in their patients receiving iliac crest autograft plus rhBMP-2 was 4 months, but the mean time to interbody fusion in patients who received rhBMP2 plus local autograft was only 3 months (excluding one patient in whom follow up was $<6$ months). No patient in the study published by Mummaneni, et al., had heterotopic foraminal bone.

Suk, et al., ${ }^{16}$ compared clinical outcomes and fusion rates in 87 patients treated with one- or two-level posterolateral fusion and bilateral or unilateral pedicle screw instrumentation. The fusion rates and clinical outcomes were equivalent for one- or two-level unilateral and bilateral pedicle screw instrumentation. Suk, et al., concluded that unilateral is as effective as bilateral pedicle screw fixation in one- or two-level posterolateral lumbar spine fusions. Furthermore, Kabins, et al., ${ }^{7}$ found that the fusion results and clinical outcomes for unilateral and bilateral pedicle screw instrumentation with VSP screws in patients undergoing L4-5 autogenous posterolateral grafting were nearly identical.

By preserving one normal facet and placing instrumentation in the pedicles on the approach side, patients in our study had rigid anterior and posterior column constructs.
Nevertheless, this system may not be as rigid as that provided by bilateral pedicle screw instrumentation. Chen and colleagues ${ }^{1}$ used an in vitro porcine model to study the biomechanical stability of one- and two-cage posterior LIF with unilateral or bilateral pedicle screw instrumentation. Although they found that constructs using two interbody cages and bilateral pedicle screw instrumentation were the stiffest ones, unilateral pedicle screw instrumentation combined with one interbody cage was a stiffer construct than the intact group without instrumentation, except in compression. Chen, et al., concluded that unilateral pedicle screw fixation with cage implantation is sufficient to maintain stability for fusion in the posterior LIF procedure.

\section{Conclusions}

The limitations of this study are its small sample size, short follow-up period, and the selected nature of the cohort. We intend to build on our database and perform follow-up evaluations in these patients over a period of several years. We await the results of other investigators studying the efficacy of unilateral percutaneous pedicle screw instrumentation in the minimally invasive TLIF procedure.

Minimally invasive TLIF in which unilateral pedicle screw instrumentation is used can be an excellent choice for selected patients with symptomatic DDD or a recurrent disc herniation. Preserving the contralateral musculature, ligaments, and tendons leaves the patient with a stronger axial trunk, which enables a faster and more com- 


\section{Unilateral percutaneous pedicle screws for minimally invasive TLIF}

plete recovery. The unilateral approach allows for decreased blood loss, decreased operating time, lower risk of intraoperative complications, earlier mobilization with less pain, earlier discharge, and earlier return to work.

\section{Disclosure}

Dr. Mobasser is a consultant and instructor for Medtronic Sofamor Danek. Dr. Beringer has no financial ties to disclose.

\section{References}

1. Chen HH, Cheung HH, Wang WK, et al: Biomechanical analysis of unilateral fixation with interbody cages. Spine 30: E92-E96, 2005

2. Foley KT, Gupta SK, Justis JR, et al: Percutaneous pedicle screw fixation of the lumbar spine. Neurosurg Focus 10 (4): E10, 2001

3. Foley KT, Langston TH, Schwender JD: Minimally invasive lumbar fusion. Spine 28:S26-S35, 2003

4. German JW, Foley KT: Minimal access surgical techniques in the management of the painful lumbar motion segment. Spine 30:S52-S59, 2005

5. Harms JG, Rollinger H: Die operative Behandlung der Spondylolisthese durch dorsale Aufrichtung und ventrale Verblockung. Z Orthop 120: 343-347, 1982

6. Harris BM, Hilibrand AS, Savas PE, et al: Transforaminal lumbar interbody fusion: the effect of various instrumentation techniques on the flexibility of the lumbar spine. Spine 29: E65-E70, 2004

7. Kabins MB, Weinstein JN, Spratt KF, et al: Isolated L4-L5 fusions using the variable screw placement system: unilateral versus bilateral. J Spinal Disord 5: 39-49, 1992

8. Lowe TG, Tahernia AD, O'Brien MF, et al: Unilateral transforaminal posterior lumbar interbody fusion (TLIF): indications, technique, and 2-year results. J Spinal Disord Tech 15:31-38, 2002
9. McKay B, Sandhu HS: Use of recombinant human bone morphogenetic protein-2 in spinal fusion applications. Spine 27: S66-S85, 2002

10. Mummaneni PV, Pan J, Haid RW, et al: Contribution of recombinant human bone morphogenetic protein-2 to the rapid creation of interbody fusion when used in transforaminal lumbar interbody fusion: a preliminary report. Invited submission from the Joint Section Meeting on Disorders of the Spine and Peripheral Nerves, March 2004. J Neurosurg Spine 1: 19-23, 2004

11. Mummaneni PV, Rodts GE: The mini-open transforaminal lumbar interbody fusion. Neurosurgery 57:256-261, 2005

12. Prolo DJ, Oklund SA, Butcher M: Toward uniformity in evaluating results of lumbar spine operations. A paradigm applied to posterior lumbar interbody fusions. Spine 11:601-606, 1986

13. Rosenberg WS, Mummaneni PV: Transforaminal lumbar interbody fusion: technique, complications, and early results. Neurosurgery 48:569-575, 2001

14. Salehi SA, Tawk R, Ganju A, et al: Transforaminal lumbar interbody fusion: surgical technique and results in 24 patients. Neurosurgery 54: 368-374, 2004

15. Sihvonen T, Herno A, Paljarvi L, et al: Local denervation atrophy of paraspinal muscles in postoperative failed back syndrome. Spine 18:575-581, 1993

16. Suk KS, Lee HM, Kim NH, et al: Unilateral versus bilateral pedicle screw fixation in lumbar spinal fusion. Spine 25: 1843-1847, 2000

17. Thomsen K, Christensen FB, Eiskjaer SP, et al: 1997 Volvo Award winner in clinical studies. The effect of pedicle screw instrumentation on functional outcome and fusion rates in posterolateral lumbar spinal fusion: a prospective, randomized clinical study. Spine 22:2813-2822, 1997

Manuscript received December 19, 2005.

Accepted in final form February 8, 2006.

Address reprint requests to: Jean-Pierre Mobasser, M.D., Indianapolis Neurosurgical Group, 8333 Naab Road, Suite 250, Indianapolis, Indiana 46260. email: jmobasser@ingmdgroup.com. 